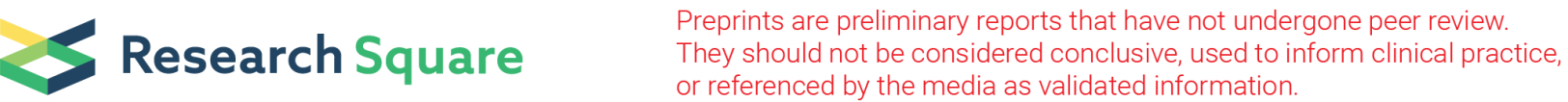

\section{PET-CT Standard uptake value predict bevacizumab- containing chemotherapy response and prognosis for unresectable colorectal cancer liver-limited metastasis}

\author{
Yang Lv \\ Zhongshan Hospital Fudan University \\ QingYang Feng \\ Zhongshan Hospital Fudan University \\ WenTao Tang \\ Zhongshan Hospital Fudan University \\ YuQiu Xu \\ Zhongshan Hospital Fudan University \\ SongBin Lin \\ Zhongshan Hospital Fudan University \\ YiHao Mao \\ Zhongshan Hospital Fudan University \\ Peng Zheng \\ Zhongshan Hospital Fudan University \\ LiangLiang Yang \\ Zhongshan Hospital Fudan University \\ GuoDong He \\ Zhongshan Hospital Fudan University \\ JianMin Xu ( $\nabla$ xujmin@aliyun.com ) \\ Zhongshan Hospital Fudan University
}

Research article

Keywords: Colorectal cancer liver metastasis; Standard uptake value; bevacizumab; prognosis

Posted Date: September 24th, 2019

DOI: https://doi.org/10.21203/rs.2.12132/v2

License: (9) This work is licensed under a Creative Commons Attribution 4.0 International License.

Read Full License 
Page 2/15 


\section{Abstract}

Background: Standard uptake value (SUV) is an indicator of tumor metabolic response. In this paper, we aim to explore the value of SUV on unresectable colorectal cancer liver-limitedmetastasis (CRLM) patients receiving bevacizumab-containing chemotherapy. Method: This study was performed retrospectively. A total of 185 CRLM patients between April 2011 to December 2015 with complete clinical data were included in this study. All enrolled patients were assigned into two treatment cohorts (bevacizumab plus chemotherapy cohort and chemotherapy only cohort). All clinical variables, and various PET/CT parameters were statistically compared with progression-free survival (PFS) and overall survival (OS). Primary and Metastatic tumor SUV were selected for analysis. Results: Among the 185 patients, 101 patients received chemotherapy plus bevacizumab ( beva cohort ), 84 patients only received chemotherapy ( CMT cohort). Baseline characteristics of two cohorts showed no statistical difference $(P>0.05)$. Primary SUV level was correlated with primary tumor size, while metastatic SUV was statistically correlated with metastatic tumor number and tumor size $(P=0.000)$. Primary lesion, metastatic lesion SUV and elevation of SUV demonstrated prognostic role for OS $(P<0.05)$. SUV gap were statistically associated with optimal response in beva cohort $(P=0.03)$ and no-PD status in CMT cohort $(P=0.019)$, respectively. After multivariate analysis, elevated SUV is an independent risk factor for OS $(P=0.000)$. Besides, elevation of SUV between metastatic and primary lesion can be a predictive factor for bevacizumab survival benefit. Conclusion: PET-CT scan is important for CRLM patients. Our study demonstrated that an elevation of SUV was a better prognostic and predictive marker for CRLM patients.

\section{Introduction}

Colorectal cancer (CRC), as one of the most common malignancies of digestive gastrointestinal tract[1], still accounts for approximately one thirds of the estimated new digestive cancer cases and incidence is continuously evolving [2]. Approximately $20 \%$ to $25 \%$ of CRC patients have liver metastases at the time of initial diagnosis and overall prognosis of patients with colorectal cancer liver metastasis (CRLM) is poor. For management of CRLM, recent development of chemotherapy regimens combined with targeted agents demonstrated decent results[3]. Bevacizumab, a humanized murine anti-human vascular endothelial growth factor (VEGF) monoclonal antibody, was approved for patients with advanced CRLM in combination with chemotherapy[4]. Many studies demonstrated that treatment with bevacizumab as compared with supportive care alone improved median survival[5].

For assessing therapeutic responses, the most prevalent way of assessing drug response is to measure volume changes in tumor dimensions based on radiographic criteria such as the Response Evaluation Criteria in Solid Tumors (RECIST)[6]. However, assessing the tumor load with RECIST-defined ORR (objective response rate) may be not enough to assess response for bevacizumab with an antiangiogenic mechanism of action[7]. Thus more accurate response evaluation as a supplementary method during early course of treatment will be more valuable for patients to receive alternative therapies as soon as possible. In this issue, another evaluation method named computed tomography (CT) morphology criteria has been developed $[8,9]$ and had a significant association with overall survival. 
As CRLM is not common, baseline tests such as computed tomography (CT) and magnetic resonance imaging (MRI) play important roles in treatment selection. In addition, fluorodexyglucose positron emission tomography/computed tomography (FDG PET/CT) is used to detect CRLM and extra-hepatic metastasis and is now widely used in oncologic evaluation. Bipat et al. reported a meta-analysis showing that PET/CT has greater sensitivity in detecting liver metastases than other noninvasive imaging modalities[10]. PET/CT is a powerful and popular tool for imaging tumors as well as to assess the quantity in tumor metabolism during therapy[11]. Assessment of standard uptake value (SUV) is widely adopted for glucose metabolism. Clinical value of SUV applied in prognosis has been explored in many cancers including CRC[12]. However, especially in patients with CRLM, studies reported to data concerning PET response to bevacizumab or chemotherapy are relatively small in terms of sample size. Thus in this study, from two cohorts of CRLM patients receiving chemotherapy with or without bevacizumab, we explored clinical value of early PET/CT scan to facilitate prediction of treatment response and survival outcomes in CRLM patients.

\section{Method And Materials}

\section{Patients and treatment modality}

Patients from April 2011 to December 2015 with unresectable CRLM was eligible into this study if the baseline PET/CT scan and SUV of both primary and metastatic site were available. Exclusion criteria included a history of significant hemoptysis, Eastern Cooperative Oncology Group (ECOG) score larger than 1, a history of thrombotic or hemorrhagic disorders. Inclusion Written informed consent was obtained by each patient (Figure 1). The study was approved by the Ethical Committee of Zhongshan Hospital, Fudan University. For the treatment protocol, patients received either chemotherapy (CMT) plus bevacizumab or CMT alone[13, 14].

The follow-up period was as follows: first follow-up was performed within 2-3 months after the baseline and subsequent follow-up cycles usually ranged from 3 to 6 months or even shorter which depended on the clinical situations and (or) tumor relapse or metastasis being suspected. Data of overall survival (OS) and relapse/metastasis time were also documented. Duration of OS was calculated from the date of baseline to the last follow-up or tumor-specific death. Progression free survival (PFS) was determined from the date of diagnosis to the date of progression under the regular follow-up.

\section{Data acquisition of ${ }^{18} \mathrm{~F}-\mathrm{FDG}-\mathrm{PET} / \mathrm{CT}$}

18-FDG PET/CT scans were obtained with a PET/CT scanner (Discovery VCT, GE Healthcare, Milwaukee, Wisconsin, USA). All patients fasted for at least 6 hours before examination. The serum glucose just before PET/CT scanning was less than $10 \mathrm{mmol} / \mathrm{dL}$. Patients were scanned at 50-60 minutes after the intravenous (i.v.) injection of F-FDG (4.44MBq/ kg), typically, patients were scanned from the base of the skull to the mid-thigh. Locations of lesions (Primary and metastatic lesions) were recorded and maximal standardized uptake values (SUVmax) of the lesions were measured. 


\section{Imaging analysis}

All the enrolled patients were subjected to multiphasic CT at the time of the initial evaluation (baseline status). The image diagnostic reports were all assessed through a double-blinded manner; Radiologist were blinded to pathologic results, patient treatment, and outcomes. Response was evaluated using CT morphologic criteria, assigning each metastasis to 1 of 3 groups (Table S1), Morphologic response criteria were defined as optimal if the metastasis changed from a group 3 or 2 to a 1 , incomplete if the group changed from 3 to 2 , and none if the group had not changed or increased[9]. Another evaluation criterion was RECIST. Figure $\mathbf{S} 1$ demonstrated the specific response status of two evaluation options. Routinely, the changes of all the patients in the post-treatment phase were evaluated from enhanced highresolution (HR) CT with less than 5-mm slices.

\section{Statistics}

Based on baseline characteristics, gender and other clinical data in both cohorts (CMT versus beva cohort) were compared by Chi-square test; age, tumor size, SUVmax values, and follow-up period were compared by Student's t-tests. PFS was assessed using Kaplan-Meier method, and survival curves were compared using a log-rank test. OS analyses were carried out using Kaplan-Meier method and results were compared using a log-rank test. A multivariable Cox proportional hazards model predicting OS was performed using backward stepwise selection. Risk factors were expressed as the hazard ratio [HR, 95\% confidence interval $(\mathrm{Cl})$ ]. Statistical significance was defined as $P$ value less than 0.05 . The discrimination of prognostic model was determined by Harrell concordance index (C-index) as an index of model performance. Higher C-index values indicate better discrimination.

\section{Results}

\section{Baseline clinical characteristics of two cohorts' patients after cases selection}

From April 2011 to December 2015, a total of 185 patients with unresectable CRLM were enrolled in our study, including 101 patients receiving first line chemotherapy plus bevacizumab (beva cohort), 84 patients receiving first-line CMT (CMT cohort). Their age range was 24-81 years and their median age was 59 years. The medians for size, CEA (carcinoembryonic antigen), CA199 (Carbohydrate antigen 199), primary SUVmax and metastatic SUVmax were $4.1 \mathrm{~cm}, 28.0 \mathrm{ng} / \mathrm{L}, 91.4 \mathrm{ng} / \mathrm{L}, 11.6$ and 9.9, respectively. Details of other clinical data were demonstrated in Table 1. Table 1 also showed that there is no difference between two groups for gender, age, primary site, ECOG score, number of metastatic site and distribution of liver metastasis.

\section{Correlation between SUV and clinical characteristics}

11.6 of primary lesion SUV, 9.9 of liver metastatic lesion were regarded as the cut-off value of primary and metastatic SUV (median value), respectively. Besides, metastatic SUV which is larger than primary lesion SUV was regarded as elevation of SUV. Table 2 demonstrated Chi-square test of clinical correlation 
between SUV and clinical characteristics. Higher primary SUV was significantly correlated with larger primary tumor size $(P=0.000)$, while metastatic SUV was related with the status of number of metastases and metastatic tumor size $(P=0.000)$. No statistical difference was observed among different Age level, gender group, primary sites, CEA, CA199 level, distribution of liver metastasis $(P>0.05)$.

\section{SUV was clinically correlated with treatment response}

Details of treatment response were listed in Table S2, overall objective response rate (ORR) and optimal response rate was $41.6 \%$ and $25.9 \%$, respectively. For different treatment protocol cohorts, CMT patients demonstrated a higher rate of progressive disease $(P D)$ rate than beva cohort patients $(P=0.014)$; there is no difference between two cohorts for partial response (PR) and stable disease (SD). Furthermore, to evaluate next efficacy of RECIST and morphologic status on prognosis, among the 185 patients, those with optimal response by morphologic criteria had better overall survival than patients with incomplete or no response in beva cohort, with median overall survival of 37 months ( $95 \% \mathrm{Cl}, 28.8-39.2$ months) and 25 months ( $95 \% \mathrm{Cl}, 21.4-28.6$ months), respectively ( $P<0.001$, Figure S2 (A)). In contrast, response by RECIST was not associated with an improvement in survival in this group (Figure S2 (C)). In addition, Figure S2 (D) also demonstrated that there is no statistically significant difference for duration of OS between optimal group and no optimal group in CMT cohort $(P>0.05)$, while RECIST criteria was more suitable for evaluating response in CMT cohort $(P<0.05$, Figure $S 2(B))$. To analyze correlation between SUV and treatment response, details of different treatment cohort have been constructed to demonstrate the statistical correlation of separately primary lesion SUV, metastatic lesion SUV and SUV gap between metastatic and primary lesion (Table 3). Compared to primary and metastatic lesions SUV, the gap was determined to have a significant relation with treatment response in both cohorts through different evaluation criteria.

\section{PET-CT SUV were significantly related with PFS and OS}

Totally, primary SUV was not a significant prognostic marker for PFS (median survival months: 7.0 months versus 6.0 months, $P=0.38$, hazard ratio $[\mathrm{HR}]=1.142,95 \% \mathrm{Cl}: 0.846-1.604)$. In contrast, higher metastatic lesion SUV ( $P=0.01)$ and elevation of SUV $(P=0.008)$ were regarded as a significant factor for PFS. In terms of OS, patients with higher primary lesion SUV had worse survival outcome than patients with lower primary SUV (median survival months: 33.0 months versus 21.0 months, $P=0.01, H R=1.799$, 95\% Cl: 1.150-2.816), while metastatic lesion SUV was also regarded as a prognostic factor for OS (median survival months: 35.0 months versus 19.0 months, $\mathrm{P}<0.001$, $\mathrm{HR}=2.512,95 \% \mathrm{Cl}: 1.605-3.932$ ). Besides, elevation of SUV between metastatic and primary lesion was demonstrated to be a significant OS-related factor with higher $\mathrm{HR}(\mathrm{P}<0.001, \mathrm{HR}=3.330,95 \% \mathrm{Cl}$ : $2.126-5.213)$. To further determine the prognostic efficacy of primary, metastatic SUV and SUV elevation, C-index of three OS-related prognostic models were evaluated. The performance of elevated SUV model was statistically better than primary SUV (C-index: 0.688 versus $0.638, P<0.001)$ and metastatic SUV level (C-index: 0.688 versus 0.568 , $\mathrm{P}<0.001)$. Details were shown in Figure 2. 
Furthermore, data of all patients were included in univariate and multivariate analysis, which was demonstrated in Table 4. From multivariable analysis of OS, CEA level $(P=0.031$, hazard ratio $[H R]=1.461$; 95\% confidential interval [Cl]: 1.063-1.795), CA199 level ( $P=0.029, \mathrm{HR}=1.806 ; 95 \% \mathrm{Cl}$ : 1.062-3.072), SUV elevation status $(P=0.000, H R=2.863 ; 95 \% C l=1.645-4.981)$ and metastatic tumor size $(P=0.012$, $\mathrm{HR}=1.631 ; 95 \% \mathrm{Cl}=1.211-2.087)$ were regarded as independent risk indicators for tumor OS.

\section{PET-CT SUV as a predictive factor for bevacizumab therapy in CRLM}

As the PET SUVmax value was often a reflection of metabolic ability on the hepatic and primary lesions, an elevated metastatic SUV may reflect a more progressive tumor metabolic ability. To confirm this hypothesis, we created another two internal controlled groups: high risk group (with elevated SUV) and low risk group (without elevated SUV). Among high risk group, our data showed that patients receiving the CMT plus bevacizumab has reached a longer duration of PFS (median survival months: 7.0 months versus 5.4 months) and OS (median survival months: 25.0 months versus 18.0 months) than patients receiving CMT only $(P<0.05)$; similar results cannot be reached in the low risk cohort, demonstrating that patients with an elevated SUV should receive a combination treatment of CMT and bevacizumab. Details were shown in Figure 3.

\section{Discussion}

Currently, development of clinical imaging devices, surgical techniques, chemotherapeutic agents have been making CRLM curable. Beyond oncogenic RAS status, bevacizumab was recommended as the effective component of first-line treatment strategy for CRLM[15]. Besides, exploration of survival benefit has become more important for treatment option selection. As many studies reported utility of radiologic and nuclear medicine, contrast of pre-treatment and post-treatment radiologic status obtained from CT and MRI was considered as the optimal process for determining response and evaluating prognosis. Based on this, RECIST has been developed and reported as the popular choice for differentiating the response[6, 7].

In addition to RECIST, a novel evaluation criteria called CT morphology criteria based on status of overall attenuation, tumor-liver interface and peripheral rim of enhancement has been reported to be more sensitive than RECIST for evaluating CRLM bevacizumab response[9]. In our study, we performed two treatment response evaluation criteria for both cohorts. The former method, proposed within RECIST, allows that response might be actually calculated comparing 2 different lesions based on tumor volume at baseline and after treatment; this contrasts with a common rule of dimensional response assessment that calls for comparing the same lesion before and after treatment. Thus in this study we also explored the response through CT morphology criteria. Our results firstly demonstrated that RECIST criteria was more sensitive than CT morphology criteria in CMT cohort, while CT morphology criteria showed preferable prognosis-related performance in beva cohort.

PET-CT SUV, as an indicator reflecting metabolic activity of tumor lesion, has also been reported and considered as a prognostic factor in many studies[16-18]. However, currently there is no evidence that 
SUV was correlated with bevacizumab treatment response. In this study, we found that primary lesion SUV was statistically correlated with primary tumor size, while metastatic SUV level was correlated with liver metastatic tumor size and tumor number. Furthermore, in our study, primary SUV and metastatic SUV have demonstrated important role for survival outcome stratification. Patients with higher SUV showed a worse survival outcome. Elevation of SUV was also proved to be related with optimal response in CT morphology criteria and PD response in RECIST criteria, determining the prediction role of baseline SUV on CRLM lesions. However, in our study, statistical difference of PFS was not shown in different primary SUV level. Since bevacizumab therapy was targeted for VEGF, PD of traditional RECIST criteria may not enough to be a study end-point in this group patients. This results were also consistent with many other studies[9]. Even ESMO guideline in 2016 has advised radiologists to pay special attention to the treatment effects if VEGF-targeting antibody bevacizumab is a component of the therapy regimen[13].

Besides, owing to reflection character of SUV on metabolic activity, we hypothesized that a baseline elevation of SUV between hepatic lesion and primary lesion may effectively affect the prognosis of CRLM patients. To further validate the hypotheses, three different SUV models were constructed with respect to clinical utility of primary lesion SUV, metastatic lesion SUV and SUV gap. Among three models, elevation of SUV showed a more promising prognostic performance with a significantly better C-index (0.688). Also, univariate and multivariate analysis of OS proved the prognostic role of SUV elevation indirectly $(P=0.000)$.

Nonetheless, the actual and definitive advantage of baseline PET/CT is that it is capable of quantifying and differentiating between primary and metastatic tissue. Using this quantification method evaluating the glucose uptake, PET/CT may improve preoperative risk stratification and optimize outcomes[19]. Thus we divided our enrolled patients into two cohorts based on SUV elevation status (low risk cohort and high risk cohort). CRLM patients in high risk cohort receiving combination therapy of bevacizumab plus CMT had a better survival outcome than their CMT counterparts, demonstrating that an elevation of SUV could be an important factor determining the optimal treatment choice. In contrast, on low risk cohort, similar results were not showed. This may be a reflection of such a phenomenon that tumor lesion with a higher level of metabolism may consist of abundant vessels with positive expression of VEGF receptor, thus those patients with higher SUV may benefit from the VEGF monoclonal antibody therapy.

There are some limitations of this study, including the retrospective design of the study, which may include selection bias. We believe that well-designed statistical criteria can alleviate this problem. A large scale prospective, randomized controlled study may be warranted to strongly determine the prognostic value of PET-CT. Another limitation is that our study only focused on the CRC live metastasis. Some CRC patients with extra-hepatic metastasis were excluded in this study because bias caused by the anatomical variety can influence the statistical results. Besides, it has been reported in some studies neoadjuvant chemotherapy was derived as statistically significant risk factor affecting prognosis for CRC liver metastasis, but we could not and did not reveal the correlation. But we believe that further wellorganized study would focus on this viewpoint. 
In this paper, we adapted CT morphology criteria and RECIST criteria to evaluate treatment response in CRC liver metastasis and found that status of CT morphology criteria is more sensitive evaluating the bevacizumab-containing chemotherapy response. Elevation of SUV obtained from baseline PET-CT scan between metastatic and primary lesion is statistically related with optimal treatment response. Furthermore, elevation of SUV is also statistically related with PFS and OS and demonstrated a better prognostic predictive performance. Besides, CRC liver metastasis patients with elevated SUV receiving bevacizumab-containing chemotherapy had a better survival outcome than their chemotherapy counterparts.

\section{Declarations}

\section{Ethics approval and consent to participate}

Written informed consent was obtained by all the patients. The study protocol followed the ethical guidelines of the Declaration of Helsinki and was approved by the Ethical Committee of Zhongshan Hospital of Fudan University.

\section{Consent for publication}

We have obtained consent to publish from the participant to report individual patient data.

\section{Availability of data and material}

The datasets used and/or analysed during the current study are available from the corresponding authors on reasonable request.

\section{Competing interests}

The authors declare no conflicts of interest for the publication of this manuscript.

\section{Funding}

This work was supported by National Natural Science Foundation of China (Grant No. 81602040, 81702302 and 81472228), Clinical science and technology innovation project of Shanghai (SHDC12016104) and Shanghai Science and Technology Committee Project (17411951300). The funding bodies had no role in the design of the study and collection, analysis, and interpretation of data and in the writing of the manuscript.

\section{Authors' contributions}

Y.L, QY.F and WT.T collected, analyzed and interpreted the patient data. and Y.L was a major contributor in writing the manuscript. JM.X and GD.H were the corresponding authors in this manuscript and were responsible for the results of this study. YQ.X, SB.L, YH.M, P.Z and LL.Y provided the research ideas and guidance. 
Acknowledgements

This manuscript has not been submitted to any other journal and is not currently being considered by another journal for publication. We Thank all the doctors and nurses during the treatment process.

Disclosure statement

The authors declare no conflicts of interest for the publication of this manuscript.

\section{References}

1. Siegel RL, Miller KD, Fedewa SA, Ahnen DJ, Meester RGS, Barzi A, Jemal A: Colorectal cancer statistics, 2017. CA Cancer J Clin 2017, 67(3):177-193.

2. Nishihara R, Ogino S, Chan AT: Colorectal-cancer incidence and mortality after screening. $N$ Engl $J$ Med 2013, 369(24):2355.

3. Cremolini C, Antoniotti C, Lonardi S, Aprile G, Bergamo F, Masi G, Grande R, Tonini G, Mescoli C, Cardellino GG et al: Activity and Safety of Cetuximab Plus Modified FOLFOXIRI Followed by Maintenance With Cetuximab or Bevacizumab for RAS and BRAF Wild-type Metastatic Colorectal Cancer: A Randomized Phase 2 Clinical Trial. JAMA Oncol 2018, 4(4):529-536.

4. Hurwitz H, Fehrenbacher L, Novotny W, Cartwright T, Hainsworth J, Heim W, Berlin J, Baron A, Griffing $\mathrm{S}$, Holmgren $\mathrm{E}$ et al: Bevacizumab plus irinotecan, fluorouracil, and leucovorin for metastatic colorectal cancer. N Engl J Med 2004, 350(23):2335-2342.

5. Simkens LH, van Tinteren H, May A, ten Tije AJ, Creemers GJ, Loosveld OJ, de Jongh FE, Erdkamp $\mathrm{FL}$, Erjavec $\mathrm{Z}$, van der Torren AM et al: Maintenance treatment with capecitabine and bevacizumab in metastatic colorectal cancer (CAIRO3): a phase 3 randomised controlled trial of the Dutch Colorectal Cancer Group. Lancet 2015, 385(9980):1843-1852.

6. Sosna J: Is RECIST Version 1.1 Reliable for Tumor Response Assessment in Metastatic Cancer? Radiology 2018:182179.

7. Pierson C, Grinchak T, Sokolovic C, Holland B, Parent T, Bowling M, Arastu H, Walker P, Ju A: Response criteria in solid tumors (PERCIST/RECIST) and SUVmax in early-stage non-small cell lung cancer patients treated with stereotactic body radiotherapy. Radiat Oncol 2018, 13(1):34.

8. Jubb AM, Harris AL: Biomarkers to predict the clinical efficacy of bevacizumab in cancer. Lancet Oncol2010, 11(12):1172-1183.

9. Chun YS, Vauthey JN, Boonsirikamchai P, Maru DM, Kopetz S, Palavecino M, Curley SA, Abdalla EK, Kaur $\mathrm{H}$, Charnsangavej $\mathrm{C}$ et al: Association of computed tomography morphologic criteria with pathologic response and survival in patients treated with bevacizumab for colorectal liver metastases. JAMA 2009, 302(21):2338-2344. 
10. Niekel MC, Bipat S, Stoker J: Diagnostic imaging of colorectal liver metastases with CT, MR imaging, FDG PET, and/or FDG PET/CT: a meta-analysis of prospective studies including patients who have not previously undergone treatment. Radiology 2010, 257(3):674-684.

11. Gollub MJ, Akhurst T, Markowitz AJ, Weiser MR, Guillem JG, Smith LM, Larson SM, Margulis AR: Combined CT colonography and 18F-FDG PET of colon polyps: potential technique for selective detection of cancer and precancerous lesions. AJR Am J Roentgenol 2007, 188(1):130-138.

12. de Geus-Oei LF, Vriens D, van Laarhoven HW, van der Graaf WT, Oyen WJ: Monitoring and predicting response to therapy with 18F-FDG PET in colorectal cancer: a systematic review. J Nucl Med 2009, 50 Suppl 1:43S-54S.

13. Van Cutsem E, Cervantes A, Adam R, Sobrero A, Van Krieken JH, Aderka D, Aranda Aguilar E, Bardelli $A$, Benson $A$, Bodoky $G$ et al: ESMO consensus guidelines for the management of patients with metastatic colorectal cancer. Ann Oncol2016, 27(8):1386-1422.

14. Yoshino T, Arnold D, Taniguchi H, Pentheroudakis G, Yamazaki K, Xu RH, Kim TW, Ismail F, Tan IB, Yeh $\mathrm{KH}$ et al: Pan-Asian adapted ESMO consensus guidelines for the management of patients with metastatic colorectal cancer: a JSMO-ESMO initiative endorsed by CSCO, KACO, MOS, SSO and TOS. Annals of Oncology 2018, 29(1):44-70.

15. Van Cutsem E, Cervantes A, Adam R, Sobrero A, Van Krieken JH, Aderka D, Aranda Aguilar E, Bardelli $A$, Benson $A$, Bodoky $G$ et al: ESMO consensus guidelines for the management of patients with metastatic colorectal cancer. Ann Oncol 2016, 27(8):1386-1422.

16. Bundschuh RA, Martinez-Moller A, Ziegler SI, Schwaiger M, Scheidhauer K: Misalignment in PET/CT: relevance for SUV and therapy management. Nuklearmedizin 2008, 47(2):N14-15.

17. Duan Y, Yu LJ, Lu PO, Wang WZ: [Correlation between SUV of (18)F-FDG PET-CT and the expression of GLUT1, MVD and Ki67 in non-small cell lung cancer]. Zhonghua Zhong Liu Za Zhi 2008, 30(10):764-767.

18. Mattes MD, Moshchinsky AB, Ahsanuddin S, Rizk NP, Foster A, Wu AJ, Ashamalla H, Weber WA, Rimner A: Ratio of Lymph Node to Primary Tumor SUV on PET/CT Accurately Predicts Nodal Malignancy in Non-Small-Cell Lung Cancer. Clin Lung Cancer 2015, 16(6):e253-258.

19. Shim JR, Lee SD, Han SS, Lee SJ, Lee DE, Kim SK, Kim SH, Park SJ, Oh JH: Prognostic significance of (18)F-FDG PET/CT in patients with colorectal cancer liver metastases after hepatectomy. Eur $J$ Surg Oncol 2018, 44(5):670-676.

\section{Figures}


Total patient group

Department of General Surgery

Zhongshan Hospital, Fudan University

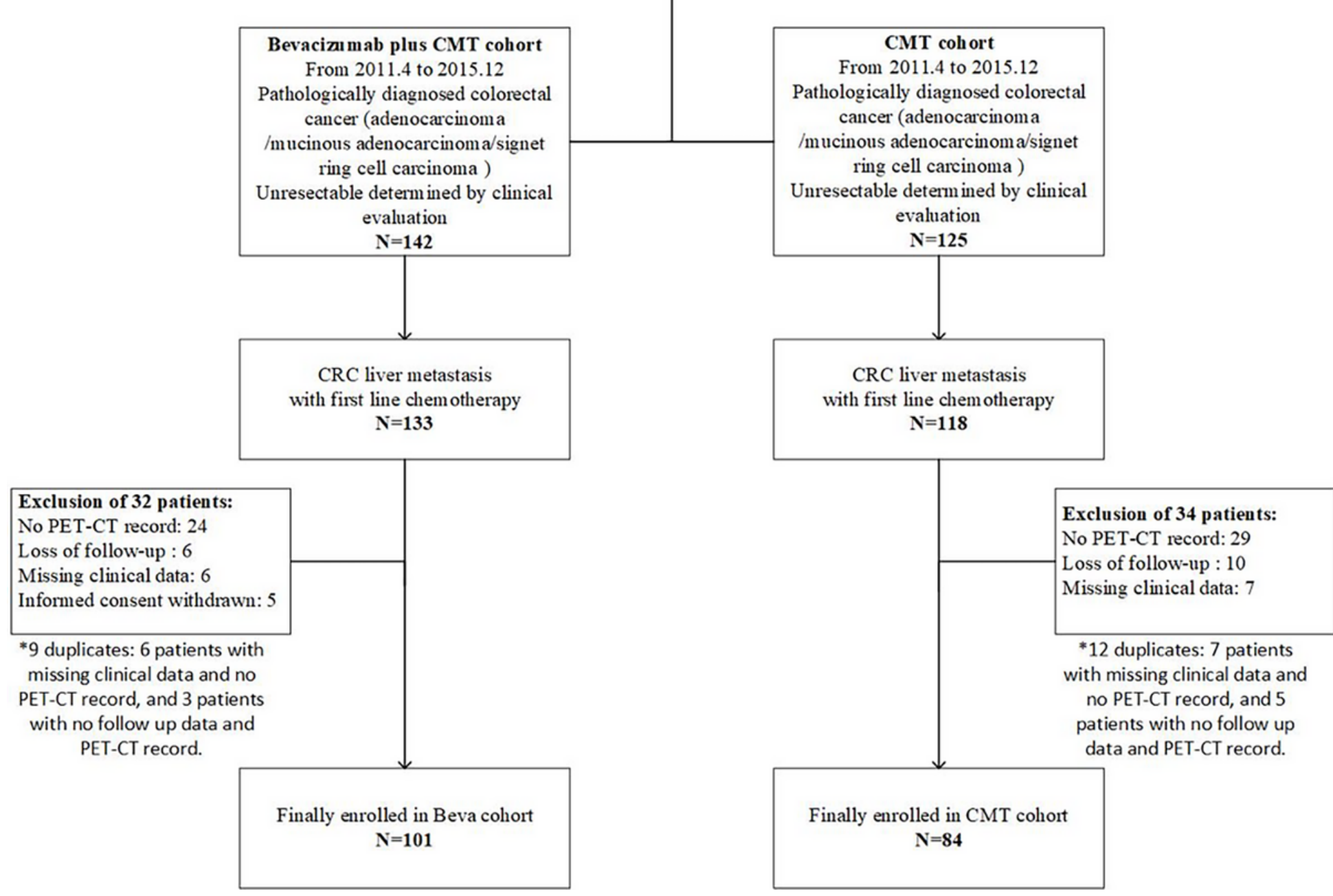

\section{Figure 1}

Flow diagram of colorectal cancer liver metastasis patients enrolled from our institution. 

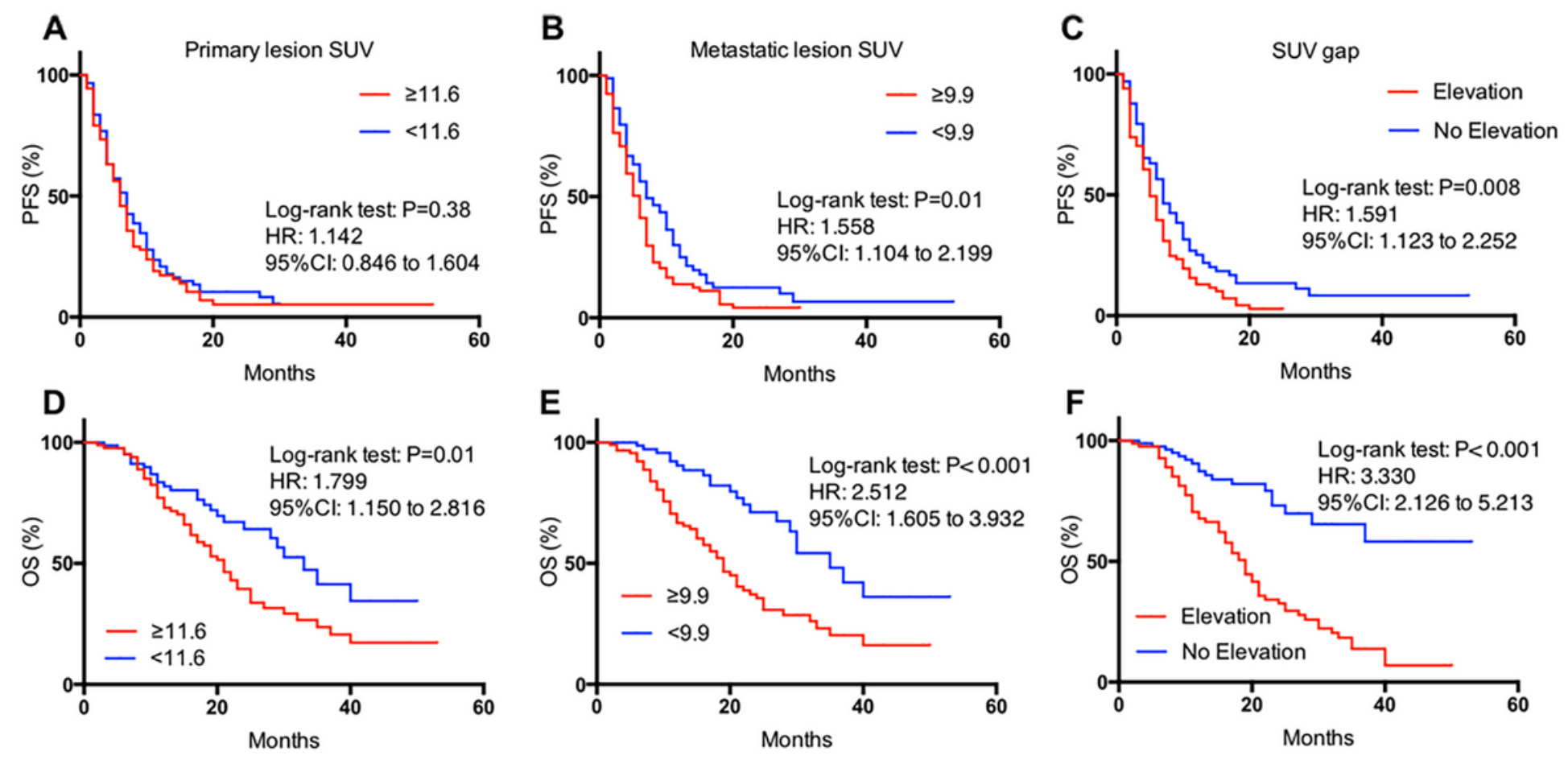

Figure 2

Kaplan-Meier progression-free survival and Overall survival curves for different primary lesion SUV level ( $A$ and $D$ ), metastatic lesion SUV level ( $B$ and $E$ ) and SUV elevation ( $C$ and $F$ ) in CRLM patients. HR, hazard ratio. 

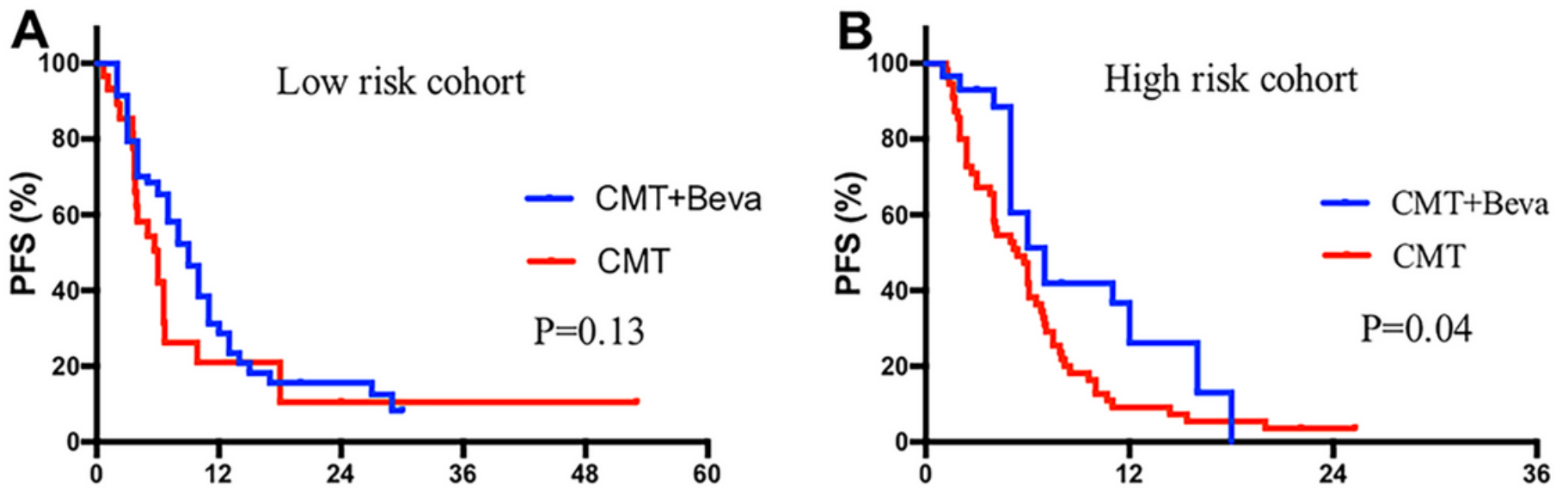

No. at risk Months

No. at risk

Months

$\begin{array}{lccccc}\text { Beva } 72 & 12 & 6 & 3 & 0 & 0 \\ \text { CMT } 29 & 5 & 2 & 1 & 1 & 0\end{array}$
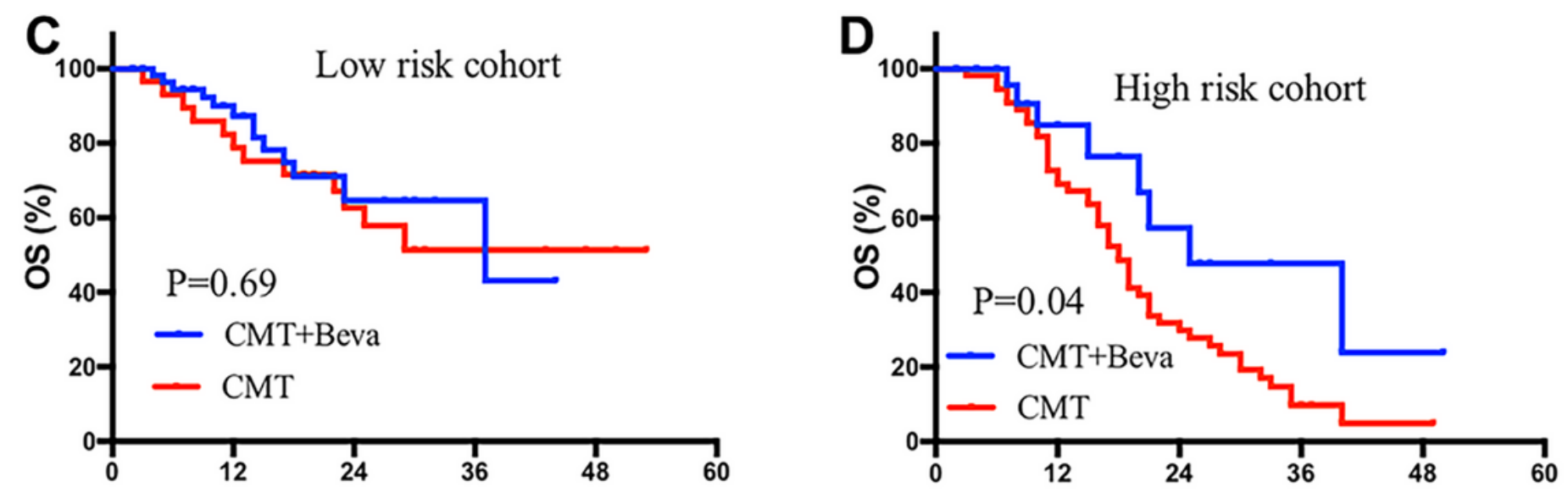

No. at risk

Months

No. at risk

Months

$\begin{array}{llllll}\text { Beva } 72 & 33 & 11 & 3 & 0 & 0 \\ \text { CMT } 29 & 23 & 13 & 6 & 2 & 0\end{array}$

$\begin{array}{cccccc}29 & 11 & 7 & 2 & 1 & 0 \\ 55 & 40 & 16 & 4 & 1 & 0\end{array}$

Figure 3

Kaplan-Meier progression free survival ( $A$ and $B$ ) and overall survival ( $C$ and $D$ ) curves for patients with different SUV gap level receiving chemotherapy or chemotherapy plus bevazunimab.

\section{Supplementary Files}

This is a list of supplementary files associated with this preprint. Click to download.

- Table3.pdf

- Table4.pdf

- Table1.pdf

- Supplementfile.pdf 
- Table2.pdf

Page 15/15 